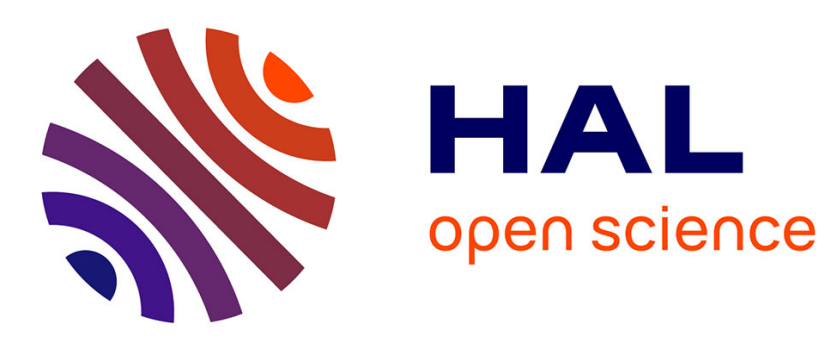

\title{
Efficacité des techniques de conservation de la semence de verrat
}

\author{
M. Paquignon, J. Bussière, F. Bariteau
}

\section{To cite this version:}

M. Paquignon, J. Bussière, F. Bariteau. Efficacité des techniques de conservation de la semence de verrat. Productions Animales, 1988, 1 (4), pp.271-280. hal-00895841

\section{HAL Id: hal-00895841 \\ https://hal.science/hal-00895841}

Submitted on 1 Jan 1988

HAL is a multi-disciplinary open access archive for the deposit and dissemination of scientific research documents, whether they are published or not. The documents may come from teaching and research institutions in France or abroad, or from public or private research centers.
L'archive ouverte pluridisciplinaire HAL, est destinée au dépôt et à la diffusion de documents scientifiques de niveau recherche, publiés ou non, émanant des établissements d'enseignement et de recherche français ou étrangers, des laboratoires publics ou privés. 
INRA Prod. Anim., 1988, 1 (4), 271-280 M. PAQUIGNON, J. BUSSIERE*,
F. BARITEAU

Institut technique du Porc M.N.E. 149, rue de Bercy 75595 Paris Cedex 12 *INRA Station expérimentale d'Insémination Artificielle 86480 Rouillé

\section{Efficacité} des techniques de conservation de la semence de verrat

\section{L'insémination artificielle porcine est de plus en plus pratiquée par les éleveurs eux-mêmes. Ces inséminations sont réalisées soit à partir de la collecte des verrats à la ferme, soit à partir de semence provenant d'un centre de production. L'utilisation de cette méthode de reproduction a nécessité la mise au point de techniques de conservation du sperme afin de maintenir son pouvoir fécondant pendant un temps suffisamment long.}

Depuis la création, en 1957, du premier centre à Loudéac (Côtes du Nord), l'insémination artificielle porcine à partir de semence produite par les centres s'est assez peu développée : 48500 IA en 1971, 72000 en 1980 et 81000 en 1987. Cependant la manière dont elle est perçue par les éleveurs a sensiblement évolué depuis quelques années. Considérée à l'origine comme un outil de

\section{Résumé}

Cet article fait le point sur la technologie de préparation de la semence en vue de sa conservation sous forme liquide ou congelée.

Pour la conservation de la semence à l'état liquide, plusieurs dilueurs sont proposés pour maintenir son pouvoir fécondant pendant plusieurs jours. Afin d'obtenir la fertilité et la prolificité les plus élevées possibles, il apparaît nécessaire, dans l'état actuel de nos connaissances, d'utiliser la fraction totale de l'éjaculat et de diluer la semence récoltée dans le milieu BTS, de facon à obtenir $3 \times 10^{9}$ spermatozoïdes dans un volume total de $100 \mathrm{ml}$ pour une insémination artificielle. Dans ces conditions, le pouvoir fécondant du sperme peut être maintenu jusqu'au $3^{\mathrm{e}}$ jour après la récolte sans baisse de la fertilité et de la prolificité.

En ce qui concerne la conservation à l'état congelé, plusieurs techniques sont proposées. La qualité de la semence après dégel varie en fonction des dilueurs de congélation et de décongélation utilisés ainsi que des technologies de préparation et de conditionnement de la semence. L'examen en microscopie électronique, après cryosubstitution, des spermatozoïdes et de leur milieu environnant montre que l'état de leur déshydratation au moment de la congélation est un facteur important pour le maintien de la qualité de la semence après dégel. Cette étude montre également qu'un des problèmes de la technique de congélation intervient au moment de la décongélation tout au moins en ce qui concerne la qualité des acrosomes. Si on considère l'ensemble des inséminations réalisées dans les 10 dernières années, il n'apparaît aucune différence entre le conditionnement en pailles et en pastilles en ce qui concerne le taux de mise bas et la prolificité. Cependant, d'autres facteurs, comme le moment de l'insémination, l'inséminateur et le verrat, peuvent influencer les résultats de fertilité. On peut estimer que par rapport au sperme frais, la congélation entraîne une fertilité inférieure de 20 à 30 points \% et une baisse de prolificité de 1 à 3 porcelets. dépannage au service des petits élevages, un certain nombre d'éleveurs voient maintenant dans l'insémination artificielle, en plus de son rôle de diffusion de la semence des meilleurs géniteurs, une technique destinée à faciliter la conduite d'élevage des truies en bandes.

C'est ainsi que l'insémination par des techniciens spécialisés de la semence provenant des centres de production a alors fait place à l'insémination par les éleveurs eux-mêmes après expédition de la semence dans les élevages. Ce mode de mise en place représentait $3 \%$ et $27 \%$ des inséminations réalisées en 1971 et en 1980, il en représente près de $90 \%$ en 1987. C'est ainsi que se développe aussi depuis quelques années l'insémination pour les éleveurs à partir de la collecte des verrats à la ferme : 50000 IA en 1983 et environ 200000 en 1987.

On peut estimer à l'heure actuelle que le nombre total de truies inséminées par les éleveurs et les inséminateurs atteint environ $12 \%$ du cheptel alors que dans les pays nordiques comme le Danemark, la Finlande, la Norvège et la Hollande ce chiffre atteint 30 à $40 \%$ du cheptel.

En pratique, compte-tenu du fait que l'expédition de la semence chez l'éleveur implique des délais d'acheminement assez longs et que, dans le cadre de l'élevage des truies en bandes, les éleveurs doivent pouvoir disposer d'une semence de bonne qualité pour inséminer les truies d'un même groupe dont les chaleurs peuvent être étalées sur plusieurs jours, il est indispensable que les spermatozoïdes gardent leur aptitude à féconder les cufs pendant un temps suffisamment long afin d'obtenir une fertilité et une prolificité acceptables. Pour cela, la mise au point de techniques de conservation bien adaptées a été nécessaire. 
L'objet de cet article est de décrire l'efficacité des deux techniques actuellement disponibles: la conservation de la semence sous forme liquide pendant quelques jours et la conservation sous forme congelée, en principe sans limite de temps.

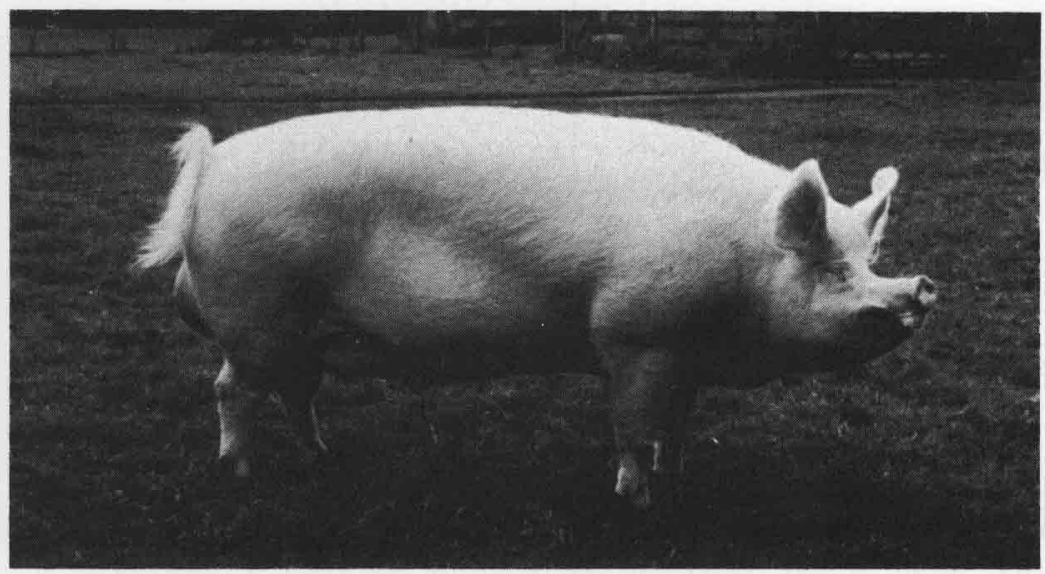

\section{1 / Conservation à l'état liquide}

Après la collecte, les spermatozoïdes mélangés au seul plasma séminal ont une faible durée de vie. L'incorporation d'un milieu susceptible de prolonger leur survie et de maintenir leur fécondance est donc indispensable. Pour être efficace, il doit fournir l'énergie nécessaire pour assurer le bon fonctionnement et le maintien de l'intégrité des cellules, avoir un système tampon s'opposant aux fortes variations de $\mathrm{pH}$, éviter le développement des germes microbiens et présenter un équilibre ionique satisfaisant.

\section{1 / Les dilueurs}

\section{a / Composition}

De nombreux dilueurs ont été utilisés pour la conservation de la semence à l'état liquide. Leur composition (Tableau 1) présente des différences notables qui entraînent des variations importantes dans la valeur de leur $\mathrm{pH}$ et de leur pression osmotique.

Cinq d'entre eux : IVT (du Mesnil du Buisson et al 1958), BL1 (Pursel et al 1973a), BTS (solution initialement utilisée pour la décongélation) (Pursel et Johnson 1975a), GUELPH, KIEV, MERCK ou VAROHM (Haeger et Mackle 1971), MODENE (Moretti 1979) sont essentiellement des solutions salines qui diffèrent entre elles par leurs teneurs en glucose, citrate de sodium, bicarbonate de sodium et par la présence ou l'absence de chlorure de potassium, d'EDTA (acide éthylène diamine tétraacétique) et d'acide citrique.

Le glucose, mieux assimilé par les spermatozoïdes que le fructose, est la meilleure source d'énergie et le seul sucre utilisé dans ces dilueurs. Il contribue aussi au maintien de l'équilibre osmotique du milieu. Le citrate de sodium, le bicarbonate de sodium et le Tris sont les seuls tampons retenus pour la semence de verrat.

L'EDTA, contenu dans la plupart de ces dilueurs, jouerait un rôle de stabilisateur du volume cellulaire (Bredderman et Foote 1971). Par ses propriétés chélatrices, il préviendrait la peroxydation des phospholipides membranaires néfaste pour le maintien du pouvoir fécondant des spermatozoïdes (Shannon et Curson 1972). Afin de mieux apprécier l'intérêt de sa présence dans les dilueurs, nous avons comparé des échantillons de sperme $\left(3 \times 10^{9} \mathrm{spz}\right)$ dilués dans $100 \mathrm{ml}$ d'un milieu contenant des quantités différentes d'EDTA (0-1,85-3,7 g/l). Les résultats montrent que l'addition d'EDTA accroît significativement la survie des spermatozoïdes après 8 jours de stockage et 5 heures d'incubation à $+37^{\circ} \mathrm{C}$ ainsi que le pouvoir fécondant du sperme et la survie embryonnaire (tableau 2).

L'albumine sérique bovine, contenue dans le dilueur ZORLESCO (Gottardi et al 1980), par ses capacités d'absorption des produits du métabolisme des spermatozoïdes et des bactéries (Bamba et Sone 1981), permet de mieux préserver la survie des spermatozoïdes (Paquignon et al 1980a).

Tous les éléments sont dissous dans un diluant constitué, la plupart du temps, par de l'eau distillée. Celle-ci peut être remplacée par de l'eau déionisée sans que le taux de conception et la mortalité embryonnaire n'en soient affectés (Diehl et Stewart 1980). Cependant, il convient d'être prudent quant à la source d'eau utilisée car, dans une expérimentation ayant pour but de proposer une technique de dilution simple aux

Tableau 1. Composition chimique des différents dilueurs utilisés pour la conservation de la semence à l'état liquide.

\begin{tabular}{|l|c|c|c|c|c|c|}
\hline \multirow{2}{*}{ PRODUITS (g) } & \multicolumn{7}{c|}{ DILUEURS } \\
\cline { 2 - 7 } & IVT & BL1 & BTS & GUELPH & MODENE & ZORLESCO \\
\hline Glucose & 3 & 29 & 37 & 60 & 27,5 & 11,5 \\
Gitrate de $\mathrm{Na} 2 \mathrm{H}_{2} \mathrm{O}$ & 19,9 & 10,0 & 6,0 & 3,7 & 6,9 & 11,7 \\
Bicarbonate de Na & 2,1 & 2,0 & 1,25 & 1,2 & 1,0 & 1,8 \\
EDTA & - & - & 1,25 & 3,7 & 2,3 & 2,1 \\
KCl & 0,4 & 0,3 & 0,75 & - & - & - \\
Sulfanilamide & 3,0 & - & - & - & - & - \\
TRIS & - & - & - & - & - & 6,5 \\
Acide citrique & - & - & - & - & 2,9 & 4,1 \\
Albumine sérique bovine & - & - & - & - & - & 5,0 \\
Cystéine & - & - & - & - & - & 0,08 \\
Eau distillée (ml) & 1000 & 1000 & 1000 & 1000 & 1000 & 1000 \\
\hline
\end{tabular}

D'autres dilueurs, le SCK 7 (Taylor 1976) et le MRA (Perez Lys 1984) ont été proposés mais leur composition n'est pas connue. 
Tableau 2. Effet de l'addition d'EDTA dans le milieu de dilution sur le pouvoir fécondant du sperme après 3 à 8 jours de conservation.

\begin{tabular}{|ccc|}
\hline $\begin{array}{c}\text { EDTA } \\
\text { (g/litre) }\end{array}$ & $\begin{array}{c}\text { Taux de } \\
\text { gestation (1) } \\
(\%)\end{array}$ & $\begin{array}{c}\text { Taux de } \\
\text { survie embryonnaire (2) } \\
(\%)\end{array}$ \\
\hline $\mathbf{0}$ & $52,3(86)_{\mathrm{a}}$ & $61,4_{\mathrm{a}}$ \\
1,85 & $70,0(90)_{\mathrm{b}}$ & $70,1_{\mathrm{b}}$ \\
3,7 & $63,6(88)_{\mathrm{ab}}$ & $68,1_{\mathrm{b}}$ \\
\hline
\end{tabular}

(1) mesuré par abattage à 60 jours de gestation

(2) rapport entre le nombre d'embryons et le nombre de corps jaunes.

() Nombre de femelles inséminées.

Les valeurs non suivies verticalement de la même lettre sont significativement différentes $(\mathrm{P}<0,05)$.

Tableau 3. Caractéristiques de la semence de verrat après stockage à $+15^{\circ} \mathrm{C}$ pendant 7 jours en présence de Dibekacin (d'après Sone 1982).

\begin{tabular}{|lcc|}
\hline & Dibekacin & $\begin{array}{c}\text { Streptomycine } \\
\text { + pénicilline }\end{array}$ \\
\hline Nombre d'échantillons & 74 & 15 \\
Motilité(\%) & $75,4_{\mathrm{a}}$ & $37,3_{\mathrm{b}}$ \\
Acrosomes normaux(\%) & $82,7_{\mathrm{a}}$ & $52,6_{\mathrm{b}}$ \\
Echantillons avec bactéries(\%) & $21,6_{\mathrm{a}}$ & $73,3_{\mathrm{b}}$ \\
Nombre de bactéries/ml(log) & 3,2 & 4,8 \\
pH & 6,8 & 6,4 \\
\hline
\end{tabular}

Les pourcentages non suivis horizontalement de la même lettre sont significativement différents $(\mathrm{P}<0,05)$

éleveurs, la comparaison de l'efficacité de l'eau distillée à celle d'une eau minérale du commerce montre qu'avec cette dernière, après 3 jours de conservation, le pourcentage de spermatozoïdes mobiles est plus faible (53\% contre $61 \%$ respectivement pour l'eau minérale et l'eau distillée).

A ces dilueurs sont ajoutés des antibiotiques. Ils limitent la prolifération des micro-organismes, dont la production d'éléments toxiques est néfaste pour la qualité de la semence. Généralement, on utilise une combinaison de pénicilline $\mathrm{G}(1000000 \mathrm{UI} / \mathrm{l})$ et streptomycine $(1 \mathrm{~g} / \mathrm{l})$ qui fournit un spectre assez large d'activité antibactérienne. D'autres antibiotiques (dibekacin, amikacin et gentamicin) utilisés à des concentrations beaucoup plus faibles et avec un spectre d'activité beaucoup plus large sont encore plus efficaces, surtout lorsque la semence est fortement contaminée à la collecte (tableau 3).

\section{b / Efficacité}

L'efficacité de ces dileurs peut être appréciée par le pourcentage de spermatozoïdes mobiles et à acrosomes normaux et par leur pouvoir fécondant après conservation.

Comparé à l'IVT qui fut l'un des tous premiers dilueurs utilisés pour la conservation du sperme, le dilueur BL1 assure un pourcentage de spermatozoïdes mobiles plus élevé durant le stockage de la semence (Bariteau et al 1976). Avec le dilueur GUELPH, leur survie n'est que très légèrement améliorée comparée à celle obtenue avec le dilueur BL1 (Paquignon et al 1979). Actuellement, les meilleurs pourcentages de spermatozoïdes mobiles et à acrosomes normaux après une longue conservation sont obtenus avec le dilueur BTS (Pursel 1983, Aalbers et al 1983, Almid et al 1984), (tableau 4).

La qualite de la semence evolue aussi avec la durée de conservation. Le pourcentage de spermatozoïdes mobiles décroît très rapidement dès le deuxième jour de conservation avec les dilueurs IVT et BL1. Par contre, il est à peu près stable avec les autres dilufurs jusqu au 3 " jour après la récolte puis décroît avec l'augmentation de la durée de conservation (Bariteau et al 1976, Paquignon et al 1979, Gottardi et al 1980, Almid et al 1984). Il en est de même de l'évolution de la qualité des acrosomes. Ainsi, une étude en microscopie électronique montre que, 10 à 15 minutes après dilution dans Le GUELPH, 11,2 \% des spermatozoïdes ont des acrosomes anormaux. Leur membrane est soulevée et, chez quelques spermatozoïdes, il y a disparition complète de la membrane de l'acrosome et de son contenu. Le pourcentage d'acrosomes gonflés

Tableau 4.Evaluation de la qualité de la semence après 7 jours de conservation dans différents dilueurs (d'après Pursel 1983).

\begin{tabular}{|lcccc|}
\hline \multirow{2}{*}{ Critères } & \multicolumn{4}{c|}{ Dilueurs } \\
\cline { 2 - 5 } & BL1 & BTS & GUELPH & MODENE \\
\hline Spermatozoïdes mobiles $(\%)$ & $28_{\mathrm{a}}$ & $68_{\mathrm{b}}$ & $39_{\mathrm{c}}$ & $59_{\mathrm{d}}$ \\
Acrosomes normaux $(\%)$ & $63_{\mathrm{a}}$ & $80_{\mathrm{b}}$ & $78_{\mathrm{b}}$ & $63_{\mathrm{a}}$ \\
\hline
\end{tabular}

Les pourcentages non suivis horizontalement d'une même lettre sont significativement différents $(\mathrm{P}<0,05)$.

La proportion de spermatozoïdes mobiles décroît très vite après 3 jours de conservation à l'état liquide. 
s'accroît après 24 heures et devient très important après 6 jours de stockage $(15,6 \%$ et $54,2 \%$ respectivement, Courtens et Paquignon 1985).

\section{c / Résultats de fertilité}

Le pouvoir fécondant des spermatozoïdes, défini comme leur aptitude à féconder des cufs et à engendrer des embryons viables, évolue aussi en fonction des dilueurs et de la durée de conservation.

Le dilueur IVT largement utilisé pendant plusieurs années gardait le pouvoir fécondant de la semence à un haut niveau seulement pendant deux jours : le jour de la récolte (J0) et le lendemain (J1) en doublant la dose d'insémination au moment de l'IA (une dose contient $3 \times 10^{9} \mathrm{spz}$ dans $100 \mathrm{ml}$ de dilueur). Comparé à ce dilueur, le BL1 a permis d'accrôitre d'une journée la durée de conservation du sperme (J0, J1, J2) en utilisant une seule dose en J0 et J1 et deux doses en J2 (Bariteau et al 1977). Avec le dilueur GUELPH et l'insémination de deux doses à partir de J2, le pouvoir fécondant est préservé plus efficacement de Jo à J2. En plus, il permet d'accroître d'une journée la durée de conservation de la semence (J0, J1, J2, J3) sans baisse de fertilité mais avec une légère chute de prolificité (Paquignon et al 1982). Le troisième jour après la récolte semble être sa limite d'utilisation. A partir de J4, la fertilité et la prolificité diminuent rapidement (Bariteau et al 1984).

Une étude entreprise au centre d'insémination artificielle de Rouillé, comparant l'efficacité des dilueurs BTS et GUELPH montre que quel que soit le jour de conservation J0 et J1 avec une dose d'insémination, J2 et J3 avec deux doses au moment de l'IA) le taux de mise bas et la prolificité obtenus avec le dilueur BTS sont toujours au moins égaux ou supérieurs à ceux obtenus avec le dilueur GUELPH (tableau 5). La prolificité reste sensiblement la même avec les deux dilueurs jusqu'à $\mathrm{J} 2$, par contre à J3 elle est plus élevée avec le dilueur BTS qu'avec le dilueur GUELPH. Ainsi la semence diluée dans le BTS peut être utilisée jusqu'à J3 sans baisse brutale de la fertilité et de la prolificité. Ces résultats sont en accord avec ceux d'Aalbers et al (1984) et Almid et al (1984).
Selon leurs auteurs, les autres dilueurs (SCK7, ZORLESCO, MODENE) devraient permettre une conservation du pouvoir fécondant de J3 à J8 et même jusqu'à J12. Cependant, le dilueur GUELPH s'est avéré être supérieur au dilueur SCK7 (Paquignon et al 1980b), ainsi qu'au dilueur de MODENE (Aalbers et al 1983) et au dilueur ZORLESCO après conservation de la semence pendant deux jours (Aalbers et al 1983), trois jours (Almlid et al 1984) ou cinq jours (Meding 1982). Bien qu'étant annoncé comme devant permettre une conservation de 8-10 jours, une étude récente (Johnson et al 1988) montre que les résultats de fertilité obtenus avec le dilueur MRA ne sont pas supérieurs à ceux obtenus avec le dilueur BTS après 3 jours de conservation.

En résumé, le BTS semble être le meilleur dilueur dont on connaisse actuellement la composition pour la dilution et la conservation de la semence, que celle-ci soit prélevée à la ferme ou dans un centre de production. Il peut être utilisé jusqu'au troisième jour après la récolte sans chute brutale de la fertilité et de la prolificité. Par rapport au dilueur GUELPH, il a aussi l'avantage de ne pas mettre les spermatozoïdes en anabiose, facilitant ainsi leur observation au microscope, et il est d'un coût de fabrication moindre.

\section{2 / Technologie de préparation de la semence}

Le volume de semence collecté et les conditions de dilution sont très importants pour la conservation du pouvoir fécondant des spermatozoïdes.

Quand ils sont dilués et conservés pendant 5 jours, le taux de mise bas obtenu avec l'éjaculat entier est significativement plus élevé que celui obtenu avec la fraction riche de l'éjaculat (Paquignon et al 1982). Ces résultats confirment d'ailleurs ceux obtenus par Meding (1976) montrant que l'élimination du plasma séminal par centrifugation avant la dilution abaisse le taux de mise bas. Sachant que l'éjaculat entier diffère de la fraction riche par la production,en fin d'éjaculation, des sécrétions des vésicules séminales, on peut leur attribuer un rôle favorable dans le processus contrôlant le pouvoir fécondant des spermatozoïdes.

Tableau 5. Fertilité et prolificité de truies inséminées avec de la semence conservée dans les dilueurs Guelph et BTS.

\begin{tabular}{|c|c|c|c|c|}
\hline Dilueurs & $\begin{array}{c}\text { Jours de } \\
\text { stockage (1) }\end{array}$ & $\begin{array}{c}\text { Femelles } \\
\text { inséminées (2) }\end{array}$ & mise bas (\%) & prolificité \\
\hline \multirow{4}{*}{ GUELPH } & 0 & 688 & 71,5 & 10,9 \\
\hline & 1 & 596 & 72,5 & 10,5 \\
\hline & 2 & 122 & 73,8 & 11,0 \\
\hline & 3 & 44 & 56,8 & 9,4 \\
\hline \multicolumn{2}{|c|}{ TOTAL } & 1450 & 71,6 & 10,7 \\
\hline \multirow{4}{*}{ BTS } & 0 & 661 & 72,9 & 10,8 \\
\hline & 1 & 589 & 77,4 & 10,9 \\
\hline & 2 & 115 & 73,9 & 11,0 \\
\hline & 3 & 50 & 68,0 & 10,8 \\
\hline \multicolumn{2}{|c|}{ TOTAL } & 1415 & 74,7 & 10,9 \\
\hline
\end{tabular}

(1) Pour J0 et J1 : 1 dose de $3 \times 10^{9} \mathrm{spz}$ par insémination; pour J2 et J3:2 doses de $3 \times 10^{9} \mathrm{spz}$ par insémination.

(2) Toutes femelles confondues inséminées une ou deux fois par l'inséminateur ou l'éleveur, dans la zone du Centre d'Insémination Artificielle de Rouillé. 
Tableau 6. Evolution de la température, du pourcentage de spermatozoïdes mobiles et d'acrosomes normaux après 6 heures de stockage à différentes températures environnantes.

\begin{tabular}{|c|c|c|c|c|c|c|}
\hline \multirow{2}{*}{$\begin{array}{l}\text { Températures } \\
\text { environnantes }\end{array}$} & \multicolumn{2}{|c|}{$\begin{array}{l}\text { Températures } \\
\text { semence }\left({ }^{\circ} \mathrm{C}\right)\end{array}$} & \multicolumn{2}{|c|}{ Spz mobiles } & \multicolumn{2}{|c|}{$\begin{array}{c}\text { Acrosomes normaux } \\
(\%)\end{array}$} \\
\hline & D & $\mathbf{F}$ & D & $\mathbf{F}$ & D & $\mathbf{F}$ \\
\hline $\begin{array}{r}-5^{\circ} \mathrm{C} \\
+5^{\circ} \mathrm{C} \\
+30^{\circ} \mathrm{C}\end{array}$ & $\begin{array}{l}25 \\
25 \\
15\end{array}$ & $\begin{array}{c}0,8 \\
7,4 \\
28,2\end{array}$ & $\begin{array}{l}86,6 a \\
86,6 a \\
86,6 a\end{array}$ & $\begin{array}{l}58,8 \mathrm{~b} \\
73,3 \mathrm{~b} \\
76,1 \mathrm{a}\end{array}$ & $\begin{array}{l}97,1 \mathrm{a} \\
97,1 \mathrm{a} \\
97,1 \mathrm{a}\end{array}$ & $\begin{array}{l}84,4 b \\
90,2 b \\
95,3 a\end{array}$ \\
\hline
\end{tabular}

$\mathrm{D}=$ début et $\mathrm{F}=$ fin du stockage $\mathrm{n}=9$

Pour chaque test d'appréciation de la qualité de la semence, les valeurs non suivies horizontalement de la même lettre sont significativement différentes $(\mathrm{P}<0,05)$.

Tableau 7. Composition des principaux dilueurs de congélation.

\begin{tabular}{|c|c|}
\hline Références & Produits \\
\hline Polge et al (1970) & Glucose : 5,67 g - Jaune d'œuf : 22,5 ml - Eau distillée : 77,5 ml \\
\hline Westendorf et al (1975] & Solution de lactose $11 \%$ : $100 \mathrm{ml}$ - Jaune d'œuf : $25 \mathrm{ml}$ - OEP : 0,5\% \\
\hline Visser et Salamon (1974) & $\begin{array}{l}\text { Tris : } 250 \mathrm{mM} \text { - Fructose : } 111 \mathrm{mM} \text { - EDTA : } 15 \mathrm{mM} \\
\text { Acide citrique : } 79,5 \mathrm{mM} \text { - Jaune d'œuf : } 15 \%\end{array}$ \\
\hline Obando et al (1984) & $\begin{array}{l}\text { Tris : } 2,432 \mathrm{~g} \text { - Glycine : } 0,939 \mathrm{~g} \text { - Acide citrique : } 1,354 \mathrm{~g} \\
\text { Glucose : } 0,75 \mathrm{~g} \text { - Eau distillée : } 100 \mathrm{ml} \text { - Jaune d'œuf : } 22,5 \mathrm{ml}\end{array}$ \\
\hline Pursel et Johnson (1975a) & $\begin{array}{l}\text { Tes : } 1,2 \mathrm{~g} \text { - Tris : } 0,2 \mathrm{~g} \text { - Glucose : } 3,2 \mathrm{~g} \text { - Jaune d'œuf : } 20 \mathrm{ml} \\
\text { OEP : } 0,5 \mathrm{ml} \text { - Ajusté à } 100 \mathrm{ml} \text { avec eau distillée }\end{array}$ \\
\hline Larsson et al (1977) & $\begin{array}{l}\text { Tes : } 4,17 \mathrm{~g} \text { - Solution } \mathrm{NaOH}(0,325 \mathrm{M}): 10,5 \mathrm{ml} \\
\text { Solution } \mathrm{KOH}(0,325 \mathrm{M}): 3,5 \mathrm{ml} \text { - Solution de glucose } 5,5 \%: 10 \mathrm{ml} \\
\text { Jaune d'œuf : } 20 \mathrm{ml} \text { - Eau distillée : } 56 \mathrm{ml} \text { - OEP : } 0,5 \% .\end{array}$ \\
\hline
\end{tabular}

L'effet du taux de dilution varie en fonction du dilueur : pas d'effet pour le BL1, (30 × $10^{6}$ vs 120 $\times 10^{6} \mathrm{spz} / \mathrm{ml}$ ) par contre,avec le dilueur GUELPH le taux de mise bas obtenus après 3 ou 5 jours de conservation est plus élevé pour une concentration de $30 \times 10^{6} \mathrm{spz} / \mathrm{ml}$ que pour une concentration de $120 \times 10^{6} \mathrm{spz} / \mathrm{ml}$ (Paquignon et al 1982)

Afin d'obtenir la fertilité et la prolificité les plus élevées possible, il apparaît nécessaire, dans l'état actuel de nos connaissances, d'utiliser l'éjaculat entier et de diluer la semence dans le dilueur BTS afin d'obtenir $3 \times 10^{9} \mathrm{spz}$ dans un volume total de $100 \mathrm{ml}$.

\section{3 / Température de stockage}

Une température de stockage comprise entre $+15^{\circ} \mathrm{C}$ et $+18^{\circ} \mathrm{C}$ a généralement été proposée pour assurer la meilleure conservation de la semence. Cependant, le maintien de cette température est difficile à réaliser lors de l'expédition de la semence chez l'éleveur. Les doses sont alors soumises aux variations externes de température et la qualité de la semence en est affectée (tableau 6). Pour éviter ces variations de température aux doses de semence, différents emballages en polyester sont utilisés par les Centres d'Insémination Artificielle. La présence de flacons d'acide acétique congelé (point de fusion $+15^{\circ} \mathrm{C}$ ) dans les emballages permet de réduire la température interne de la boîte quand la température externe est élevée $\left(+30^{\circ} \mathrm{C}\right)$ mais est sans effet quand celle-ci est basse $\left(-5^{\circ} \mathrm{C}\right)$. C'est par une augmentation des capacités thermiques de l'emballage (expédition d'un nombre important de doses dans le même emballage) plutôt que par la recherche de matériaux sophistiqués que l'on pourra maintenir une température interne voisine de $+15^{\circ} \mathrm{C}$ malgré des températures externes pouvant atteindre $-10^{\circ} \mathrm{C}$ (Von Muller-Schlosser et al 1980).

\section{2 / Congélation de la semence}

Depuis les premières naissances de procelets après insémination de semence congelée (Polge et al 1970, Pursel et Johnson 1971, Crabo et Einarsson 1971), plusieurs techniques de congélation ont été proposées. Elles diffèrent entre elles par les dilueurs et les processus de congélationdécongélation.

\section{1 / Les dilueurs de congélation et décongélation}

\section{a / Dilueurs de congélation}

\section{- Composition}

Plusieurs dilueurs sont maintenant utilisés pour la congélation de la semence de verrat (tableau 7). Ils sont caractérisés par une faible force ionique et un $\mathrm{pH}$ voisin de la neutralité

Ils sont constitués des principaux éléments suivants : agent cryoprotecteur, sucre, protéine et additif. Chacun de ces éléments joue un rôle spécifique dans la protection des spermatozoïdes. 


\section{- Rôle}

Parmi un grand nombre d'agents cryoprotecteurs, seuls le glycérol, l'érythritol, le xylitol, l'adonitol, l'acétamide et le diméthylsulfoxide (DMSO) ont un rôle de protection quand ils sont additionnés au dilueur de congélation.

Le meilleur pourcentage de spermatozoïdes mobiles et le maximum d' oufs fécondés sont toujours obtenus avec le glycérol quand il est comparé aux autres agents cryoprotecteurs ou en combinaison avec eux (Visser et Salamon 1974). Ainsi, le glycérol reste le plus largement utilisé pour la congélation du sperme de verrat. Cependant, il doit être utilisé à faible concentration depuis qu'il a été montré qu'un niveau de $7 \%$ ou plus dans le dilueur réduit ou inhibe le pouvoir fécondant des spermatozoïdes (Wilmut et Polge 1974). Les raisons de cette réduction ne sont pas encore bien identifiées. Néanmoins, il a été montré que de fortes concentrations altèrent la perméabilité membranaire des spermatozoïdes (Bower et al 1973), réduisent le pourcentage d'œufs fécondés, même après insémination par voie chirurgicale (Wilmut et Polge 1972), abaissent la consommation d'oxygène (Sanford et al 1972) et réduisent la survie des spermatozoïdes après 3 heures d'incubation à $+37^{\circ} \mathrm{C}$ (Paquignon et Courot 1975b).

La réduction de la concentration de glycérol dans les dilueurs à un taux de $2 \%$ permettant d'obtenir le maximum de spermatozoïdes mobiles avec le maximum de spermatozoïdes à acrosomes normaux (Pursel et al 1978a) a été probablement l'une des principales améliorations dans la technologie de la congélation (Westendorf et al 1975, Pursel et Johnson 1975a, Paquignon et Courot 1976, Larsson et al 1977). A l'extrême, Polge (1976) conclut même que sa présence n'est pas indispensable.

Le fructose, le glucose et le lactose sont les principaux sucres additionnés aux dilueurs. Ils agissent par leur propriété osmotique et comme cryoprotecteur extracellulaire. L'effet protecteur de ces sucres dépend pour beaucoup de leur interaction avec les autres constituants du milieu, de leur concentration et du critère utilisé pour apprécier la qualité de la semence (Visser et Salamon 1974).

Le jaune d'œuf entre dans la composition de tous les dilueurs. Il contribue à améliorer le pour- centage de spermatozoïdes mobiles et à acrosomes normaux (Visser et Salamon 1974). Les mécanismes par lesquels le jaune d'œuf protège les spermatozoïdes ne sont pas bien connus ; il agirait par une moindre déshydratation cellulaire au moment de la congélation (Courtens et Paquignon 1985).

Un détergent synthétique (Orvus Es Paste: OEP) a été introduit dans certains dilueurs pour préserver l'intégrité cellulaire. Il accroît le pourcentage de spermatozoïdes mobiles et à acrosomes normaux (Graham et al 1971) et augmente le pouvoir fécondant du sperme (Pursel et al 1978b). Le mécanisme d'action de l'OEP n'est pas bien connu, mais certains résultats montrent qu'il agit en altérant la composition du jaune d'œuf plutôt que directement sur la membrane des spermatozoïdes (Pursel et al 1978b). Sa concentration optimum est de l'ordre de 1 à $2 \%$ (Westendorf et al 1975, Pursel et al 1978b).

\section{- Efficacité}

Peu d'études systématiques ont été entreprises pour comparer l'efficacité de ces dilueurs. En prenant comme critère de qualité le pourcentage de spermatozoïdes mobiles, Obando et al (1984) ont montré que le dilueur TRIS, glycine, glucose était supérieur aux dilueurs BF5 ou TES-NA-K. En réalité, la supériorité d'un dilueur sur l'autre dépend pour beaucoup du taux de dilution, du processus de refroidissement, de la méthode de congélation et des critères utilisés pour apprécier la qualité du sperme.

Paquignon et al (1986) ont montré qu'un dilueur lactose-OEP-jaune d'œuf comparé à un dilueur glucose-jaune d'œuf améliore les pourcentages de spermatozoïdes mobiles et d'acrosomes normaux après décongélation et trois heures d'incubation. Cependant, des variations importantes apparaissent en fonction du mode de conditionnement de la semence. Il apparaît que le dilueur contenant du glucose est le mieux adapté pour le maintien du pourcentage de spermatozoides mobiles lors du conditionnement en pastilles mais très peu adapté pour le conditionnement en pailles de $5 \mathrm{ml}$, alors que le dilueur lactose-OEP peut être utilisé pour tous les modes de conditionnement (pastilles ou pailles de $0,5 \mathrm{ml}$ ), en assurant toujours la meilleure protection des
Figure 1. Effet du dilueur de congélation et du mode de conditionnement du sperme sur la qualité des spermatozoïdes après dégel (d'après Paquignon et al 1986).

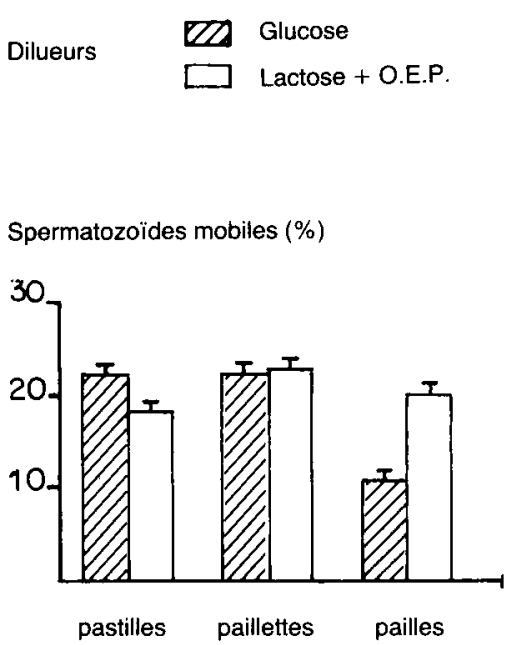

Acrosomes normaux (\%)

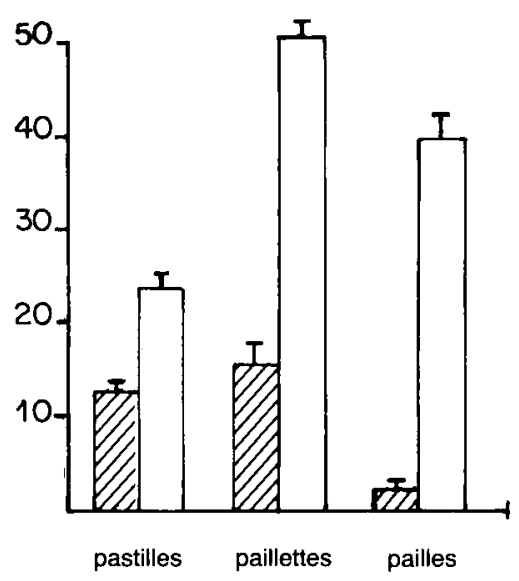


Tableau 8. Composition des solutions de décongélation.

\begin{tabular}{|l|c|c|c|c|}
\hline \multirow{2}{*}{ Produits (g) } & \multicolumn{4}{c|}{ Solution de dégel } \\
\cline { 2 - 5 } & BTS & OLEP & Hülsenberg & INRA-ITP \\
\hline Fructose & - & 5 & - & - \\
Glucose & 37 & - & 57,5 & 3 \\
Lactose & - & - & 2,5 & - \\
Citrate de $\mathrm{Na} 2 \mathrm{H}_{2} \mathrm{O}$ & 6 & - & 4,5 & 19,9 \\
EDTA & 1,25 & - & 3,5 & 3,7 \\
Bicarbonate de Na & 1,25 & 0,84 & 1,2 & 2,1 \\
$\mathrm{KCl}_{\text {Pyruvate de Na }}$ & 0,75 & 1,2 & 0,4 & - \\
CaCl & - & 5 & - & - \\
$\mathrm{MgCl}_{2}$ & - & 0,66 & - & - \\
$\mathrm{NaCl}_{\text {Eau distillée }(\mathrm{ml})}$ & - & $\mathbf{0 , 8 6 5}$ & - & - \\
& - & 3,5 & - & 1000 \\
\hline
\end{tabular}

acrosomes (Figure 1). L'examen en microscopie électronique de ces dilueurs fixés après congélation montre que le dilueur glucose présente une structure dense, homogène, avec très peu de microcristaux de glace, alors que le dilueur lactose-OEP contient des microcristaux plus gros et plus nombreux, de 0,1 à 0,5 microns (Courtens et Paquignon 1985). Ainsi, l'effet favorable du lactose-OEP par rapport au glucose pourrait résider dans une moindre déshydratation du dilueur et des cellules qu'il contient au moment de la congélation. Lors de la décongélation, étape importante pour le maintien de l'intégrité des acrosomes, les spermatozoïdes subiraient alors un moindre choc osmotique (Courtens et Paquignon 1985).

La comparaison de l'efficacité des dilueurs est très difficile car la plupart d'entre eux ont été développés comme une partie intégrante d'un processus de congélation de telle sorte qu'ils ne sont généralement pas transférables d'une méthode à l'autre.

\section{b / Solutions de décongélation}

L'utilisation du plasma séminal comme milieu de dégel a été considéré comme un élément essentiel quand cette nouvelle technologie a été proposée (Crabo et Einarsson 1971). Cependant, Larsson et Einarsson (1976) ont obtenu des fertilités équivalentes avec de la semence décongelée dans du plasma séminal, avec ou sans protéine, ou dans de l'OLEP (plasma séminal synthétique). Des dilueurs synthétiques appropriés peuvent donc remplacer entièrement ou en partie le plasma séminal. Ainsi les solutions de dégel les plus communément utilisées sont maintenant des solutions salines. Elles comprennent le BTS (Pursel et Johnson 1975a), le D8 d'Hülsenberg (Westendorf et al 1975) l'INRA-ITP (Paquignon et Courot 1976) et l'OLEP (Larsson et Einarsson 1976) (Tableau 8).

Le glucose est le principal sucre de ces solutions. Il est associé avec du lactose dans la solution Hülsenberg et remplacé par le fructose et le pyruvate de sodium dans l'OLEP. L'EDTA présent dans les milieux d'Hülsenberg, BTS et INRA-ITP, améliore la survie des spermatozoïdes après incubation (Visser et Salamon 1974). Contrairement aux dilueurs de congélation, l'efficacité des solu- tions de décongélation est maximale lorsque la concentration en électrolytes est élevée (Senegacnik et al 1980). Les solutions utilisées sont pour la plupart isotoniques ; seuls Westendorf et al (1975) adoptent une hypertonicité. Celle-ci limiterait le choc osmotique lié à la décongélation et améliorerait la qualité de la semence au dégel (Vasquez et Graham 1980).

L'effet des solutions de dégel sur la qualité de la semence et son pouvoir fécondant dépend de leur composition. Ainsi, comparée aux solutions Hülsenberg, BL1, plasma séminal et lait, la solution BTS permet d'obtenir les meilleurs pourcentages de spermatozoïdes mobiles et à acrosomes normaux (Pursel et Johnson 1975a). Cependant, la survie des spermatozoïdes durant l'incubation, le taux de gestation et la survie embryonnaire sont significativement plus élevés avec la solution INRA-ITP qu'avec la solution BTS (Paquignon et al 1977).

\section{2 / Processus de congélation et décongélation}

\section{a / Refroidissement avant congélation}

La préparation et le refroidissement ont pour but d'amener la semence de la collecte à la congélation dans les meilleures conditions de qualité. Les spermatozoïdes sont d'abord soit laissés au contact du plasma séminal avant centrifugation (Pursel et Johnson 1975a), soit dilués dans un nouveau milieu après élimination du plasma séminal suivant la centrifugation (Paquignon et al 1974, Westendorf et al 1975). Le contact des spermatozoïdes avec le plasma séminal ou un autre milieu de dilution pendant un certain temps a la particularité d'augmenter leur résistance au choc au froid. Le développement de cette résistance est une caractéristique propre du spermatozoïde. Le plasma séminal l'améliore mais ne semble pas essentiel (Pursel et al 1973b). Son rôle durant cette période n'est d'ailleurs pas encore bien défini : différentes études permettent de conclure que sa présence ou son absence ne modifie pas d'une façon appréciable la qualité de la semence (Pursel et al 1973b, Moore et Hibbit 1977). Dans le succès de ces nouvelles technologies, c'est peut-être da-

\section{Contrairement aux dilueurs de congélation, l'efficacité des solutions de décongélation est maximale lorsque la concentration en électrolytes est élevée.}


Tableau 9. Aspect qualitatif des spermatozoïdes au cours du processus de congélation-décongélation (d'après Courtens et Paquignon 1985).

\begin{tabular}{|l|c|c|c|}
\hline \multirow{2}{*}{\multicolumn{1}{|c|}{ Anomalies }} & \multicolumn{3}{|c|}{ Spermatozoïdes } \\
\cline { 2 - 4 } & $+\mathbf{5}^{\mathbf{0}} \mathbf{C}$ & Congelés & Décongelés \\
\hline Acrosomes gonflés $(\%)$ & 1,6 & 1,4 & 56,4 \\
Flagelles vacuolisés & + & +++++ & +++++ \\
\hline
\end{tabular}

+ : importance moyenne de l'atteinte des flagelles.

vantage la durée de contact et de refroidissement qui est importante. En effet, toutes les techniques de congélation donnant une fertilité satisfaisante recommandent une durée de refroidissement supérieure à 4 heures, pour amener la température de la semence de $37^{\circ} \mathrm{Cà}+5^{\circ} \mathrm{C}$ entre la collecte et la congélation. Dans la plupart des techniques proposées, l'élimination du plasma séminal par centrifugation a pour simple but d'augmenter la concentration des spermatozoïdes au moment de la congélation pour diminuer le volume de stockage.

Cette concentration doit cependant rester dans des valeurs limites raisonnables. Pour le maintien de la qualité de la semence, à l'heure actuelle, une concentration comprise entre 450 et $600 \times 10^{6}$ $\mathrm{spz} / \mathrm{ml}$ est habituellement utilisée (Westendorf $e t$ al 1975, Paquignon et Courot 1975b).

La température de la semence juste avant congélation doit être la plus basse possible. L'optimum se situe vers $4-5^{\circ} \mathrm{C}$. Dans ces conditions le taux de survie des spermatozoïdes est amélioré par rapport à une congélation de semence dont la température est comprise entre 8 et $22^{\circ} \mathrm{C}$ (Paquignon et al 1974, Westendorf et al 1975).

L'incubation du sperme avec ou sans plasma séminal, l'addition de dilueurs après l'élimination du plasma séminal, le maintien d'une haute concentration en spermatozoïdes durant le refroidissement à $+15^{\circ} \mathrm{C}$ sont les principaux points communs aux différentes technologies durant la période précédant la congélation.

\section{b / La congélation}

Au cours de la congélation proprement dite, quel que soit le mode de conditionnement de la semence, pastille ou paille, les spermatozoïdes traversent une zone de température critique dans laquelle ils sont fortement endommagés. Soixante-dix à quatre-vingt pour cent des anomalies dues à la congélation apparaissent entre 0 et $-20^{\circ} \mathrm{C}$ (Pursel et Park 1985). Ceci est la conséquence de la formation de cristaux de glace (Niwa et Taguchi 1981)

Ces dommages peuvent être réduits quand les vitesses de la congélation sont adaptées au mode de conditionnement de la semence.

Avec les premières techniques, les vitesses de congélation étaient très faibles. Dans ces conditions le pourcentage de spermatozoïdes mobiles le plus élevé était obtenu quand la concentration en glycérol était supérieure à $5 \%$ mais, dans ce cas, le pouvoir fécondant des spermatozoïdes était nul (Dalrymple et McPherson 1969). Depuis 1970, il est généralement admis que des vitesses de congélation plus élevées, obtenues lors de la congélation en pastilles (Nagase et Niwa 1964) en combinaison avec de faibles concentrations de glycérol, sont préférables pour la survie des spermatozoïdes et le maintien de leur pouvoir fécondant. Cette méthode est utilisée dans les principales techniques actuellement proposées (Pursel et Johnson 1975a, Paquignon et Courot 1975a, Larsson et al 1977). La taille des pastilles peut varier de 0,05 à $0,2 \mathrm{ml}$ sans affecter la qualité de la semence (Salamon 1973, Pursel et Johnson 1976) ; au-delà, il y a une baisse de cette qualité (Kozumplik 1978).

Les spermatozoïdes peuvent également être congelés en pailles de différents volumes. En général, la qualité de la semence obtenue après congélation en paillette de $0,5 \mathrm{ml}$ est supérieure à celle obtenue après congélation en paille de $5 \mathrm{ml}$ (Paquignon et al 1986). Cependant, pour des raisons pratiques de volume de semence à congeler, ce sont les pailles de $5 \mathrm{ml}$ qui sont utilisées (Westendorf et al 1975). Avec des volumes supérieurs le pouvoir fécondant des spermatozoïdes est faible (Larsson et al 1976).

\section{c / La décongélation}

Jusqu'à maintenant, il était difficile d'apprécier d'une façon objective, dans la chute de la qualité de la semence, la part revenant au processus de congélation de celle revenant au processus de décongélation. Une expérience récente de cryosubstitution, montrant que seulement 1 à $2 \%$ des spermatozoïdes ont des acrosomes gonflés au moment de la congélation alors que $60 \%$ d'entre eux ont la même anomalie après dégel, indique qu'un des principaux problèmes de la technologie survient au cours de la décongélation (Courtens et Paquignon 1985) (tableau 9).

Plus celle-ci est rapide, meilleure est la qualité de la semence (Pursel et Johnson 1976). C'est pourquoi des solutions de dégel, destinées à accélérer les échanges thermiques, sont utilisées pour la décongélation des pastilles (Pursel et Johnson 1976). La température de dégel est aussi un facteur de réussite, surtout pour la décongélation des pastilles mais moins pour celle des pailles (Westendorf et al 1975, Perezcanto-Fernandez 1978). Il existe donc une vitesse de décongélation optimale variant selon les techniques de congélation. Dans la pratique courante, les pastilles sont décongelées dans une solution à $50^{\circ} \mathrm{C}$ (Pursel et Johnson 1976, Paquignon et Courot 1976, Larsson et al 1977) et les pailles dans un bain d'eau chaude à $50^{\circ} \mathrm{C}$ (Westendorf et al 1975) avec une durée d'immersion qui doit être comprise entre 40 s et 50 s (Pursel et Park 1985).

\section{3 / Résultats de fertilité}

De nombreuses études ont été réalisées pour développer des tests de laboratoire pouvant prédire le pouvoir fécondant du sperme : morpholo- 
gie des acrosomes (Pursel et al 1972), décharge de GOT (Crabo et al 1972), résistance à l'incubation (Larsson 1976), pouvoir de fixation des protéines (Pavelko et Crabo 1976), contenu en ATP (Aalbers et al 1985). Cependant, aucun de ces tests ne permet de prédire la fertilité. Aussi l'ultime test pour déterminer l'efficacité d'une technique de préparation de la semence est la mesure du taux de mise bas et de la prolificité.

Si l'on considère l'ensemble des inséminations réalisées au cours des 10 dernières années, il n'apparaît aucune différence significative des taux de mise bas et de prolificité entre les deux techniques de conditionnement de la semence : pailles et pastilles (Johnson 1985) (Figure 2). Ce-

Figure 2. Comparaison de l'efficacité de la congélation en pastilles $(n=987)$ ou en pailles $(n=1727)$ sur la conservation du pouvoir fécondant du sperme (d'après Johnson 1985).

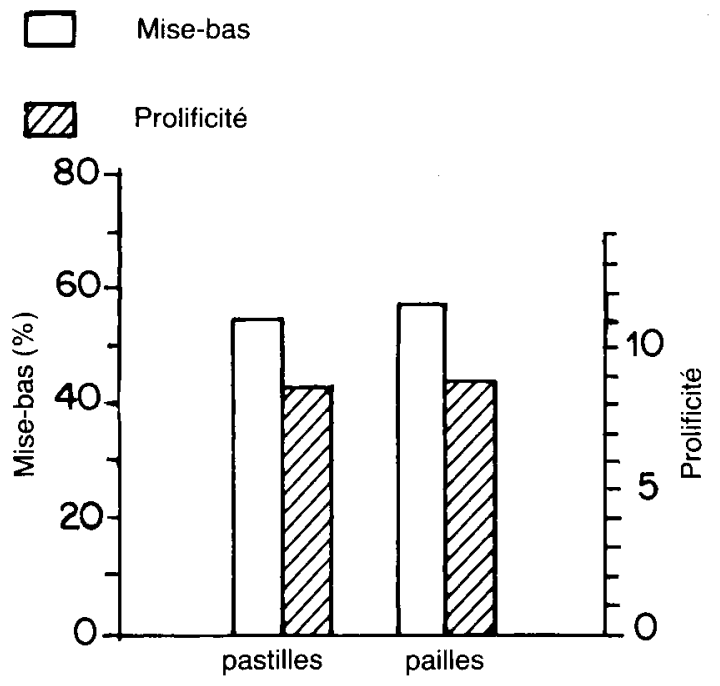

pendant, d'autres facteurs peuvent influencer les résultats de fertilité. Pour obtenir une réussite maximum, le moment de l'insémination en semence congelée doit se situer le plus près possible du moment de l'ovulation (Pursel et Johnson 1975b, Larsson 1976). Ainsi $33 \%$ contre $70 \%$ des truies deviennent gravides quand l'insémination est réalisée respectivement 12 ou 24 heures après l'immobilisation de la truie (Paquignon et al 1976). Il semble que le plus faible taux de survie des spermatozoïdes congelés par rapport aux spermatozoïdes frais soit une des raisons majeures nécessitant l'insémination de la semence congelée le plus près possible du moment de l'ovulation (Pursel et al 1978c).

Aussi la détection précise des chaleurs qui était déjà un élément important dans le succès de l'LA en sperme frais est encore plus importante pour l'IA en semence congelée. Pour augmenter les chances d'inséminer le plus près possible du moment de l'ovulation, on peut pratiquer une double insémination (Almlid et Stavne 1985), mais cette technique est coûteuse en doses de semence et en temps de travail.

De nombreuses autres études ont également montré un effet du verrat (Larsson 1976, Paquignon et al 1980c) et de la race (Johnson et al 1981) sur le taux de mise bas. Une variation entre verrats ou entre races de la survie des spermatozoïdes dans le tractus femelle peut expliquer cet effet (Larsson 1976)

D'autres facteurs, comme l'inséminateur ou la qualité de l'œestrus de la femelle, peuvent aussi influencer la fertilité (Paquignon et al 1980c). Par contre, la durée de stockage dans l'azote liquide ne semble pas affecter le pouvoir fécondant du sperme (Johnson 1985)

Toutes ces données montrent qu'il est possible d'obtenir une fertilité acceptable (environ $60 \%$ de mises-bas) après insémination en semence congelée. Toutefois, il faut remarquer qu'elle n'a pu être obtenue qu'après un contrôle très strict des conditions de son utilisation. En pratique avec la semence congelée les valeurs du taux de mise bas et de la prolificité sont environ 20 à 30 points \% et 1 à 3 porcelets inférieures à celles obtenues en sperme frais.

Ces moindres fertilité et prolificité sont certainement la conséquence d'un plus faible pourcentage d'œufs fécondés chez les truies inséminées avec du sperme congelé que chez les truies inséminées en sperme frais (Lwoff et al 1987) et d'un retard significatif $(\mathrm{P}<0,05)$ du développement des ovocytes fécondés par du sperme congelé (tableau 10), probablement lié aux modifications que subit la chromatine du noyau des spermatozoïdes au cours de la congélation (Hamamah et al 1987).
Les résultats de reproduction sont moins bons lorsqu'on utilise la semence congelée : baisse de la fertilité de 20 à 30 points et baisse de la prolificité de 1 à 3 porcelets.

Tableau 10. Evolution du stade de développemént des œufs fécondés dans les oviductes avec du sperme frais ou congelé.

\begin{tabular}{|c|c|c|}
\hline $\begin{array}{c}\text { Stade de développement des ceufs fécondés } \\
\text { (nombre de cellules) }\end{array}$ & \multicolumn{2}{|c|}{ Fpermatozoïdes } \\
Congelés \\
\hline 1 & $1120,9(30)$ & $29,5(38)$ \\
2 & $42,7(61)$ & $53,5(69)$ \\
$3-4$ & $25,9(37)$ & $13,9(18)$ \\
$>4$ & $10,5(15)$ & $3,1(4)$ \\
\hline Total & $100(143)$ & $100(129)$ \\
\hline
\end{tabular}

(1) : Pourcentage - 0 : nombre d'œufs fécondés.

(29 truies ont été inséminées dans les oviductes (un oviducte en sperme frais, l'autre en sperme congelé) et abattues 28 heures après le moment présumé de l'ovulation). 


\section{Conclusion}

Les techniques de préparation de la semence en vue de son utilisation en insémination artificielle sont efficaces aussi bien sous forme liquide que congelée.

De l'ensemble des dilueurs actuellement proposés, le BTS apparaît le mieux adapté pour la conservation de la semence à l'état liquide, au moins jusqu'au troisième jour après la récolte, sans baisse de fertilité et prolificité si au moment de l'insémination la dose de semence est doublée quand la conservation atteint le deuxième jour après la récolte. Au-delà du troisième jour après la récolte, nous n'avons pas d'indications suffisantes pour nous prononcer sur l'utilisation de ce dilueur. Pour obtenir une fertilité maximum, il est préférable que la dilution s'effectue à partir de la fraction totale de l'éjaculat plutôt qu'à partir de la seule fraction riche.

Différentes techniques de congélation ont été proposées. Dans la pratique, la technique d'insémination artificielle avec de la semence congelée n'est pas très développée et la plupart des inséminations sont réalisées avec de la semence conservée à l'état liquide. Le problème majeur du processus de congélation est le faible taux de spermatozoïdes mobiles obtenu après dégel : 20 à $40 \%$ selon les techniques. Aussi le nombre élevé de spermatozoïdes nécessaire pour obtenir une fertilité raisonnable $\left(6\right.$ à $10 \times 10^{9}$ spermatozoïdes totaux) accroît d'une façon importante le coût de ce nouveau service comparé à celui de la semence liquide. De nouveaux progrès ne pourront être réalisés qu'en approfondissant nos connaissances sur les échanges intervenant entre la cellule et son environnement durant le processus de congélation et de décongélation.

\section{Principales références bibliographiques}

(La liste complète des références utilisées est disponible auprès des auteurs)

A ALBERS J.G., JOHNSON L.A., RADEMAKER J.H.M. GROOTEN H.J.G., 1984. Use of boar spermatozoa for A.I. Fertility and morphology of semen diluted in BTS and used for insemination within 24 hrs or 25 to 48 hrs after collection. Xth Int. Congr. Anim. Reprod. A.I, Urbana, II, 180.

ALMLID T., BLICHFELDT T., STAVNE S.E., 1984. Trials with different diluting fluids for boar A.I. semen. Xth Int. Congr. Anim. Reprod. A.I. Urbana, II, 182.

BARITEAU F., BUSSIERE J., COUROT M., PAQUIGNON M., 1984. Insémination artificielle porcine. Mise en place par l'éleveur ou l'inséminateur. Bilan technique et résultats pratiques : intérêts de la méthode. Journées Rech. Porcine en France, 16, 173-180.

COURTENS I.L., PAQUIGNON M., 1985. Ultrastructure of fresh, frozen and frozen, thawed spermatozoa of the boar. First Int. Conf. Deep. Freezing Boar Semen, Uppsala, 61-87.

HAFGER O., MACKLE N., 1971. Ein Zweiphasen Verdüner in der Praxis der Schweinebesamung. Deutsch Tierarztl. Wschr., 78, 395-397.

JOHNSON L.A., AALBERS J.G., WILLEMS C.M.T., SYBESMA W., 1981. Use of boar spermatozoa for artificial insemination. I Fertilizing capacity of fresh and frozen spermatozoa in sows on 36 farms. J. Anim. Sci., 52, 1130-1136. JOHNSON L.A., 1985. Fertility results using frozen boar spermatozoa : 1970 to 1985 . First Int. Conf. Deep. Freezing Boar Semen, Uppsala, 199-222.

JOHNSON L.A., AALBERS J.G., GROOTEN H.J.G., 1988 Artificial insemination of swine : fecundity of boar semen stored in Beltsville T.S. (BTS), Modified Modena (M.M.), or MRA and inseminated on one, three and four days after collection. Zuchthyg. 23, 49-55.
LARSSON K., EINARSSON S., 1976. Fertility of deepfrozen boar spermatozoa. Influence of thawing diluents and of boars. Acta Vet. Scand., 17, 43-62.

LWOFF L., BEZARD J., PAQUIGNON M., 1987. Comparaison de technologies de conservation des spermatozoïdes de verrat. Effet sur les mécanismes de la fécondation. Journées Rech. Porcine en France. 19, 79-86

du MESNIL du BUISSON F. DAUZIER L., 1958. Maintien du pouvoir fécondant du sperme de verrat en présence de gaz carbonique. C.R. Acad. Sci., Série D, 247, 2472-2475.

PAQUIGNON M., MERGOUNIS D., COUROT M., du MESNIL du BUISSON F., 1974. Technologie de congélation de la semence de verrat : étude « in vitro ». Journées Rech. Porcine en France, 6, 71-74.

PAQUIGNON M., COUROT M., 1975b. Survie des spermatozoïdes de verrat après décongélation. Effet du rythme de collectes, de la concentration et du taux de glycérol. Ann. Biol. Anim. Bioch. Biophys., 15, 517-523.

PAQUIGNON M., DELAHAYE C., BUSSIERE J., COUROT M., 1976. Fertilité et prolificité de truies inséminées avec du sperme congelé : effet du moment de l'insémination. Journées Rech. Porcine en France, 8, 181-184.

PAOUIGNON M., DACHEUX J.L., COUROT M., 1977. Effet de différentes solutions de décongélation sur le pouvoir fécondant des spermatozoïdes de verrat. Journées Rech. Porcine en France, 9, 15-18.

PAQUIGNON M., BUSSIERE J., BARITEAU F., COUROT M. 1980c. Effect of frozen boar semen under pratical conditions of artificial insemination. Theriogenology, 14, 217226

PAQUIGNON M., BARITEAU F., BUSSIERE J., DACHEUX J.L., COUROT M., 1982. Effet du dilueur du taux de dilution et du plasma séminal sur la fertilité des truies après une longue conservation de la semence. Journées Rech. Porcine en France, 14, 85-89.

PAQUIGNON M., QUELLIER P., DACHEUX J.L., 1986 Congélation du sperme de verrat: comparaison de différents dilueurs, techniques de préparation de la semence, modes de conditionnement et températures de décongélation. Ann. Zootech., 35(2), 173-184.

POLGE C., SALAMON S., WILMUT., 1970. Fertilizing capacity of frozen boar semen following surgical insemination. Vet. Rec., 87, 424-428.

PURSEL V.G., JOHNSON L.A., SCHULMAN L.L., $1973 a$. Fertilizing capacity of boar semen stored at 15C. J. Anim. Sci., 37, 532-535.

PURSEL V.G., JOHNSON L.A., SCHULMAN L.L., 1973b. Effect of dilution, seminal plasma and incubation period on cold-shock susceptibily of boar spermatozoa. I. Anim. Sci. 35. 580-584

PURSEL V.G., JOHNSON L.A., 1975a. Freezing of boar spermatozoa. Fertilizing capacity with concentrated semen and a new thawing procedure. J. Anim. Sci, 40, 99-102. PURSEL V.G., JOHNSON L.A., 1976. Frozen boar spermatozoa : methods of thawing. J. Anim. Sci., 42, 927-932.

PURSEL V.G., SCHULMAN L.L., JOHNSON L.A., 1978a. Effect of glycerol concentration of frozen boar sperm. Theriogenology, 9, 305-312

PURSEL V.G., SCHULMAN L.L., JOHNSON L.A., $1978 \mathrm{~b}$ Effect of Orvus ES Paste on acrosome morphology, motility and fertilizing capacity of frozen thawed boar sperm. J. Anim. Sci., 47, 198-202.

PURSEL V.G., SHULMAN L.L., IOHNSON L.A., 19780 Distribution and morphology of fresh and frozen thawed sperm in the reproductive tract of gilts after artificial in semination. Biol. Reprod., 19, 69-76.

SALAMON S., 1973. Deep freezing of boar semen. III Effects of centrifugation, diluent and dilution rate, pellet volume and method of thawing on survival of spermatozoa. Aust. J. Biol. Sci., 26, 239-247.

VISSER D., SALAMON S.S., 1974. Effect of composition of tris based diluent on survival of boar spermatozoa following deep freeazing. J. Biol. Sci., 27, 485-497.

WESTENDORF P., RICHTER L., TREU H., 1975. Zur Tiefgefrierung von Ebersperma : Labor und Besamungsergebnisse mit dem Hülsenberger Pailleten Verfahren. Dtsch. Tierärztl. Wschr., 82, 261-267.

WILMUT J., POLGE C., 1974. The fertilizing capacity of boar semen stored in the presence of glycerol at 20,5 and $79^{\circ} \mathrm{C}$. J. Reprod. Fert., 38, 105-113. 